\title{
Breast sarcoidosis: 3 cases and literature review
}

\author{
Catherine Chapelon-Abric* \\ Department of Internal Medicine and Clinical Immunology, University Hospital Pitié-Salpêtrière, France
}

\begin{abstract}
Breast involvement in sarcoidosis is rare, reported in less than $1 \%$ of cases. We report 3 new cases of breast sarcoidosis and analyzed the 22 previously published cases. Breast involvement was the only manifestation of sarcoidosis in only three cases while breast sarcoidosis was the site of sarcoidosis at presentation in 12 cases. Sarcoidosis was multi-systemic in $45 \%$ of the 25 cases. Except in one case, physical examination revealed multiple breast masses without skin/nipple changes or lymphadenopathy. X-ray, ultrasound of the breasts showed multiple irregular and/or spiculated hypoechoic masses without micro calcifications. MRI showed mass with gadolinium enhancement. Treatments, rarely given for BS rather than sarcoidosis, included steroids and immunosuppressant therapy, usually showed, a good efficacy in these series.
\end{abstract}

\section{Introduction}

Sarcoidosis is a systemic granulomatous disease of unknown etiology which can affect any tissue in the body. Sarcoidosis mostly occurs in young adults, especially between 20 and 40 years [1]. This inflammatory disease is caused by alteration of cellular immune response after multiple factors i.e. environmental, occupational, and genetic and/or infections [2]. The most frequent organs involved are lungs, lymph nodes, skin, eyes and liver. Sarcoidosis breast involvement is reported in less than $1 \%$ of cases although it is probably an underestimation due to non-systematic performance of mammography $[3,4]$. Malignancy must always be considered [5-7]. Breast sarcoidosis can be diagnosed during the follow up of carcinoma $[3,6]$ or both diseases can be diagnosed concomitantly $[8,9]$. We herein report 3 new cases of breast sarcoidosis and review in depth 22 cases previously published in literature with English abstract [4-7,10-26].

\section{Case report}

\section{Case 1}

A 44-year-old Caribbean female with a multi-systemic sarcoidosis (peripheral, thoracic and abdominal lymphadenopathy; pulmonary involvement; myelopathy and intracranial involvement) was successfully treated by both prednisone $(0.5 \mathrm{mg} / \mathrm{kg} / \mathrm{d})$ and subcutaneous methotrexate $(0.3 \mathrm{mg} / \mathrm{kg} /$ week $)$. Immunosuppressive therapy was progressively reduced to obtain in 2010 a maintenance dose regimen (prednisone $5 \mathrm{mg} / \mathrm{d}$; hydroxychloroquine $400 \mathrm{mg} / \mathrm{d}$ ). In June 2015, CT scan showed stable pulmonary changes. FDG PET scan demonstrated hypermetabolic activity of mediastinal (SUV max 3.7) and axillary (SUV max 3.7) lymphadenopathy and bilateral pulmonary condensation (SUV max 7.4). Of note, there was no breast hypermetabolism. A systematic mammography revealed cystic lesions of inferior quadrants of the left breast. Breast ultrasound showed bilateral ectasia of mammary duct associated with a left dense nodular lesion which was suggestive of bilateral breast cancer (ACR4 classification). Pathology results of bilateral core biopsy showed a bilateral granulomatous inflammation consistent with sarcoidosis, without associated malignancy. The persistent pulmonary sarcoidosis activity was treated by prednisone $(5 \mathrm{mg} / \mathrm{d})$ and methotrexate $(0.2$ mg/week). In 2017, a clinical and morphologic relapse of sarcoidosis (central nervous system, pulmonary) was noted. FDG-PET-scan showed persisting bilateral breast hyper activity and abnormal pictures on echography (left hypoechoic lesion) / mammography (ill-defined mass with irregular contour) (right: ACR2; left ACR4) and MRI (breast hamartoma) (right: ACR2; left ACR3). She received for six months pulses of cyclophosphamide with no response. Then, she was treated by infliximab until March, 2018 ( $5 \mathrm{mg} / \mathrm{kg}$ ) when sarcoidosis remained quiescent with neither neurological nor pulmonary signs.

\section{Case 2}

In 1997, a 42-year-old Caribbean female presented, a severe multisystemic sarcoidosis with erythema nodosum, uveitis, severe lung infiltration, lymphadenopathy and liver involvement. Compliance to treatment revealed very poor for more than 5 years. In 2005, she started a combination with prednisone $(0.5 \mathrm{mg} / \mathrm{kg} / \mathrm{d})$, mycophenolate mofetil ( $3 \mathrm{gr} / \mathrm{d}$ ), and monthly intravenous pulses of cyclophosphamide. A partial pulmonary and hepatic response was observed. After stopping cyclophosphamide, she was treated by infliximab $(5 \mathrm{mg} / \mathrm{kg} / 8$ weeks). Six months later, infliximab was withdrawn because of a paradoxical reaction (sarcoid lesions, biological inflammatory process, more severe hepatic disorders) and the patient status worsened. FDG-PETscan showed diffuse, severe, hypermetabolic activity on thoracic, abdominal and pelvic nodes (SUX max 5.5 to 15.8), liver (SUV max 6), lymphoid facial structure (SUV max 10.5), bones (SUV max 8.1) and left breast (SUV max 5.5). Mammography was normal. Due to the discovery of that breast hypermetabolism along a multisystemic and severe sarcoidosis relapse, without any mammographic abnormality, we did not consider adequate to request a breast biopsy and supposed that the breast hyper metabolism corresponded to BS. She was treated

${ }^{\star}$ Correspondence to: Catherine Chapelon-Abric, Department of Internal Medicine and Clinical Immunology, CHU Pitié-Salpétrière, 47-83, boulevard de l'Hôpital, 75013 Paris, France, E-mail: catherine.chapelon@aphp.fr

Key words: breast sarcoidosis, breast malignancy, sarcoidosis, FDG-PET-scan

Received: August 10, 2018; Accepted: September 03, 2018; Published: September 07, 2018 
successfully by cyclophosphamide pulses with prednisone. Three years later, FDG-PET-scan showed a good persistent response on nodes, bones, and nasal localization; there was no more breast hypermetabolic activity.

\section{Case 3}

A 57-year-old Maghrebian female was admitted for multi-systemic sarcoidosis with skin (sarcoid lesion), nasal (nasal obstruction), salivary gland, joints (arthralgia), cervical nodes, stage II thoracic involvement and neurological signs (migraines). Cerebral MRI revealed meningeal gadolinium enhancement. Physical exam revealed a non-inflammatory tumefaction in the upper outer quadrant of the left breast, without skin change. The echography/mammography revealed a lesion with irregular borders. Breast MRI showed a T2 enhancement with T1 hyposignal, associated with enhancement on gadolinium images. Breast biopsy showed non-caseating giant cell granulomas without element of breast cancer. She received prednisone $(0.5 \mathrm{mg} / \mathrm{kg} / \mathrm{d})$ associated with methotrexate $(0.2 \mathrm{mg} / \mathrm{kg} /$ week $)$. After 10 years-follow-up period, she presented a neurological relapse of the sarcoidosis; however, there was no relapse of breast granuloma and no appearance of breast cancer.

\section{Literature review and discussion}

Sarcoidosis is a multi-systemic granulomatous disease of unknown etiology [1]. Breast sarcoid involvement is rarely reported and always in women [4-7,10-26]. In our sarcoidosis' series including 382 females, we observed 7 breast cancers (1.8\%) and 3 breast sarcoidosis $(0.8 \%)$, rates similar to those of the Lower's series [3]. Main characteristics of our three cases and twenty-two cases found in the literature are detailed in Tables 1 and 2 [4-7,10-26]. For the present study, we excluded cases with history of breast carcinoma and/or incidental finding of regional sarcoidosis-like reaction to the cancer in a breast removed for carcinoma as well as all differential diagnoses (Table 3). Of note, the comparative analysis revealed difficult to perform as cases were analyzed through either a pathological or radiological approach rather than clinical.

After review (Table 1) [4-7,10-26], BS appears exceptional under 30 years, mean age at BS diagnosis is 47.84 years (29-67) and in 59\% $(10 / 17)$ in black women $[5,6,11,12,16,22,23,26]$ (Cases 1 and 2). Breast involvement was the sole manifestation of sarcoidosis in only three cases $[9,17,18]$, and BS revealed sarcoidosis in $50 \%$ of cases $[4,17,23]$.The most frequent sarcoidosis localizations associated with BS in 22 cases

Table 1. Main characteristics of patients with breast sarcoidosis

\begin{tabular}{|c|c|c|c|c|c|c|c|c|}
\hline & Ethnicity & $\begin{array}{c}\text { Age } \\
\text { SS* } \\
\text { (years) }\end{array}$ & $\begin{array}{c}\text { Age } \\
\text { BS* } \\
\text { (years) }\end{array}$ & Manifestations of systemic sarcoidosis & $\begin{array}{l}\text { Positive biopsies for } \\
\text { sarcoidosis }\end{array}$ & $\begin{array}{c}\mathrm{ACE} \text { at } \\
\mathrm{BS}^{*}\end{array}$ & Treatment for sarcoidosis & $\begin{array}{c}\text { Follow up } \\
\text { of BS } \\
\text { (months) }\end{array}$ \\
\hline Ridgen B [4] & White & 22 & 29 & $\begin{array}{c}\text { Unilateral linear shadowing, negative } \\
\text { tuberculin test }\end{array}$ & Breast & ND & 0 & 48 \\
\hline Ross MJ [5] & Black & 25 & 29 & Stage I thoracic & Kweim test & ND & Prednisone & 48 \\
\hline $\begin{array}{l}\text { Gansler TS [6] } \\
\text { Case } 1\end{array}$ & Black & 31 & 31 & Stage II, lymph nodes, ocular & $\begin{array}{l}\text { Lymph node } \\
\text { Pulmonary } \\
\text { Breast }\end{array}$ & ND & ND & ND \\
\hline $\begin{array}{l}\text { Fitzgibbons PL [7] } \\
\text { Case } 1\end{array}$ & White & 70 & 65 & Stage I thoracic & Lymph node & ND & 0 & 120 \\
\hline $\begin{array}{l}\text { Fitzgibbons PL [7] } \\
\text { Case } 2\end{array}$ & White & 49 & 49 & None & Breast & ND & 0 & ND \\
\hline Banik S [10] & White & 28 & 29 & Stage I thoracic, negative tuberculin test & Kweim test & high & ND & ND \\
\hline Mc Pherson [11] & Black & 54 & 64 & $\begin{array}{l}\text { Cutaneous, superior vena cava } \\
\text { syndrome }\end{array}$ & $\begin{array}{l}\text { Kweim test, Cutaneous, } \\
\text { Lymph node, Breast }\end{array}$ & ND & 0 & 60 \\
\hline Donaldson BA [12] & Caribbean & 44 & 44 & Stage II thoracic, hypercalcemia & Kweim test & high & Steroids & ND \\
\hline Kenzel PP [13] & ND & 55 & 61 & $\begin{array}{c}\text { Stage III thoracic, negative tuberculin } \\
\text { test }\end{array}$ & Bronchial & normal & prednisone & 24 \\
\hline Gisvold JJ [14] & ND & 57 & 67 & Cutaneous, cardiac & Cutaneous & ND & ND & ND \\
\hline Harris KP [15] & ND & 53 & 53 & None & Breast & normal & 0 & 12 \\
\hline Ojeda H [15] & Black & 43 & 43 & None & Breast & ND & ND & ND \\
\hline Takahashi R [17] & Japanese & 48 & 48 & Stage I thoracic & Breast & high & ND & 25 \\
\hline Ishimaru K [18] & ND & 26 & 31 & $\begin{array}{l}\text { Stage I thoracic, cutaneous, negative } \\
\text { tuberculin test }\end{array}$ & Breast & normal & 0 & 0 \\
\hline El Khoury M [19] & ND & 50 & 50 & Stage III thoracic & Breast & ND & 0 & 0 \\
\hline Fiorucci F [20] & White & 50 & 51 & Stage II thoracic, uveitis & Breast & high & Prednisone $0.5 \mathrm{mg} / \mathrm{kg} / \mathrm{d}$ & 20 \\
\hline Nicholson BT [21] & ND & $?$ & 58 & Stage III thoracic, pituitary & Breast & ND & ND & ND \\
\hline Hermann G [22] & Black & 31 & 41 & Cutaneous & Breast & ND & 0 & ND \\
\hline Rishi M [23] & Black & 31 & 39 & $\begin{array}{l}\text { Stage I thoracic } \\
\text { Liver }\end{array}$ & Breast & ND & Prednisone & 12 months \\
\hline Panzacchi R [24] & ND & 57 & 57 & Stage II thoracic & Trans bronchial biopsy & high & Steroids & 15 \\
\hline Zujic PV [25] & ND & 54 & 54 & $\begin{array}{c}\text { Stage II thoracic } \\
\text { High calcium level }\end{array}$ & Breast & high & Prednisone & 24 \\
\hline Mason C [26] & Black & 37 & 37 & Stage II thoracic & Breast & ND & 0 & ND \\
\hline Case 1 & Caribbean & 44 & 51 & $\begin{array}{c}\text { Stage II thoracic, central nervous } \\
\text { system, salivary glands, diffuse } \\
\text { lymphadenopathy }\end{array}$ & $\begin{array}{c}\text { Bronchial } \\
\text { Lymphadenopathy } \\
\text { Salivary glands, } \\
\text { Nervous system }\end{array}$ & high & $\begin{array}{c}\text { Prednisone: } 5 \mathrm{mg} / \mathrm{d} \\
+ \text { MTX } 0.2 \mathrm{mg} / \mathrm{kg} / \mathrm{wk} ; \\
\text { cyclophosphamide; infliximab }\end{array}$ & 24 \\
\hline
\end{tabular}




\begin{tabular}{|l|c|c|c|c|c|c|c|}
\hline Case 2 & Caribbean & 42 & 58 & $\begin{array}{c}\text { Stage III thoracic, liver, ocular, } \\
\text { cutaneous, nasal diffuse } \\
\text { lymphadenopathy, poor general } \\
\text { condition }\end{array}$ & $\begin{array}{c}\text { Bronchial, liver, } \\
\text { Osteo-medullar } \\
\text { Lymph node }\end{array}$ & high & $\begin{array}{c}\text { Cyclophosphamide, } \\
\text { Prednisone: } 15 \mathrm{mg} / \mathrm{d}\end{array}$ \\
\hline Case 3 & Maghrebian & 57 & 57 & $\begin{array}{c}\text { Stage II thoracic, central nervous } \\
\text { system, ocular, nasal, salivary glands, } \\
\text { joint }\end{array}$ & Nasal & high & $\begin{array}{c}\text { Prednisone: } 50 \mathrm{mg} / \mathrm{d} \\
+\mathrm{MTX} 20 \mathrm{mg} / \mathrm{wk}\end{array}$ \\
\hline
\end{tabular}

*SS: Systemic Sarcoidosis, BS: Breast Sarcoidosis, ACE: Angiotensin Converting Enzyme, MTX: Methotrexate, ND: Not Determined

Table 2. Main characteristics of breast sarcoidosis

\begin{tabular}{|c|c|c|c|c|c|c|c|}
\hline & $\begin{array}{l}\text { Age at } \\
\text { BS }^{*} \\
\text { (years) }\end{array}$ & BS detection & Echography & Mammography & CT-scan/MRI & $\begin{array}{c}\text { Fine needle } \\
\text { aspiration } \\
\text { cytology }\end{array}$ & $\begin{array}{l}\text { Core biopsy } \\
\text { excision }\end{array}$ \\
\hline Ridgen B [4] & 29 & Right breast nodule & ND & ND & ND & ND & $\mathrm{ND} /+$ \\
\hline Ross MJ [5] & 29 & $\begin{array}{l}\text { Two irregular non tender } \\
\text { breast masses }\end{array}$ & ND & ND & ND & ND & $\mathrm{ND} /+$ \\
\hline $\begin{array}{l}\text { Gansler TS [6] } \\
\text { Case } 1\end{array}$ & 31 & Palpable nodule & ND & ND & ND & ND & $\mathrm{ND} /+$ \\
\hline $\begin{array}{l}\text { Fitzgibbons PL [7] } \\
\text { Case 1 }\end{array}$ & 65 & Palpable nodule & ND & ND & ND & ND & $\mathrm{ND} /+$ \\
\hline $\begin{array}{l}\text { Fitzgibbons PL [7] } \\
\text { Case } 2\end{array}$ & 49 & Mass & ND & ND & ND & ND & $\mathrm{ND} /+$ \\
\hline Banik S [10] & 29 & Smooth mobile lesion & Normal & Normal & ND & ND & $\mathrm{ND} /+$ \\
\hline Mc Pherson [11] & 64 & Firm breast left mass & ND & ND & ND & ND & $\mathrm{ND} /+$ \\
\hline Donaldson BA [12] & 44 & $\begin{array}{c}\text { Painless left breast masses, } \\
\text { soft, non-tender, } \\
\text { movable }\end{array}$ & ND & ND & ND & ND & $\mathrm{ND} /+$ \\
\hline Kenzel PP [13] & 61 & Mass & $\begin{array}{l}\text { Hypoechoic lesion, } \\
\text { sharply outlined } \\
\text { contours }\end{array}$ & Ill-defined mass & $\begin{array}{l}\text { Hyper intense, inhomogeneous } \\
\text { tissue, irregular contours } \\
\text { early washout phase }\end{array}$ & ND & $\mathrm{ND} /+$ \\
\hline Gisvold JJ [14] & 67 & Systematic mammography & ND & $\begin{array}{c}\text { Multiple non-calcified } \\
\text { spiculated bilateral nodules }\end{array}$ & ND & ND & $+/ \mathrm{ND}$ \\
\hline Harris KP [15] & 53 & $\begin{array}{l}\text { Breast discomfort } \\
\text { Localized nodule }\end{array}$ & Normal & $\begin{array}{c}\text { Pronounced nodularity and } \\
\text { architectural distortion }\end{array}$ & ND & ND & $+/+$ \\
\hline Ojeda H [15] & 43 & Mass & ND & ND & ND & - & $\mathrm{ND} /+$ \\
\hline Takahashi R [17] & 48 & $\begin{array}{c}\text { Painless right breast mass, } \\
\text { soft movable mass }\end{array}$ & ND & $\begin{array}{l}\text { Ill-defined mass without } \\
\text { calcification }\end{array}$ & ND & + & $\mathrm{ND} /+$ \\
\hline Ishimaru K [18] & 31 & Mass & $\begin{array}{l}\text { Small, irregular } \\
\text { hypoechoic lesion } \\
\text { surrounded by } \\
\text { echogenic rim }\end{array}$ & $\begin{array}{l}\text { Spiculated mass without } \\
\text { calcifications }\end{array}$ & $\begin{array}{l}\text { Hyper intense with irregular } \\
\text { border after gadolinium }\end{array}$ & ND & $\mathrm{ND} /+$ \\
\hline El Khoury M [19] & 50 & Palpable painless mass & Hypoechoic lesion & $\begin{array}{c}\text { Mass with speculated } \\
\text { margins. }\end{array}$ & $\begin{array}{c}\text { Intensely enhancing speculated } \\
\text { mass }\end{array}$ & ND & $+/ \mathrm{ND}$ \\
\hline Fiorucci F [20] & 51 & Palpable nodule & Hypoechoic lesion & Speculated left lesion & ND & ND & $\mathrm{ND} /+$ \\
\hline Nicholson BT [21] & 58 & Mammography & $\begin{array}{c}\text { Multiple bilateral round } \\
\text { ill-defined hypoechoic } \\
\text { mass with hyperechoic } \\
\text { rims }\end{array}$ & $\begin{array}{c}\text { Bilateral, round, ill-defined } \\
\text { equal density }\end{array}$ & ND & ND & $+/ \mathrm{ND}$ \\
\hline Hermann G [22] & 41 & Chest discomfort & ND & $\begin{array}{c}\text { Asymmetric left breast } \\
\text { density }\end{array}$ & ND & ND & $+/ \mathrm{ND}$ \\
\hline Rishi M [23] & 39 & Mammography & ND & $\begin{array}{l}\text { Left breast micro- } \\
\text { calcifications }\end{array}$ & ND & ND & $\mathrm{ND} /+$ \\
\hline Panzacchi R [24] & 57 & Mammography & ND & Suspicious eft breast nodule & ND & + & $+/+$ \\
\hline Zujic PV [25] & 54 & $\begin{array}{l}\text { Extensive skin lesion of left } \\
\text { breast; palpable induration; } \\
\text { pigmentation; axillary } \\
\text { lymphadenopathy }\end{array}$ & $\begin{array}{c}\text { Hypoechoic lesion with } \\
\text { thickened skin }\end{array}$ & $\begin{array}{l}\text { Parenchymal blurring fused } \\
\text { within the pectoral muscle }\end{array}$ & $\begin{array}{l}\text { Extensive inflammatory } \\
\text { changes of left breast; } \\
\text { infiltration of pectoral muscle; } \\
\text { enlarged left axillary lymph }\end{array}$ & ND & $+/ \mathrm{ND}$ \\
\hline Mason C [26] & 37 & Mammography & $\begin{array}{c}\text { Ill-defined hypoechoic } \\
\text { lesion }\end{array}$ & $\begin{array}{c}\text { Single dilated duct of right } \\
\text { breast }\end{array}$ & ND & + & $\mathrm{ND} / \mathrm{ND}$ \\
\hline Case 1 & 51 & Echography & $\begin{array}{l}\text { Bilateral ectasia of } \\
\text { mammary ducts and } \\
\text { dense nodular lesion }\end{array}$ & Cystic lesions & ND & ND & $+/ \mathrm{ND}$ \\
\hline Case 2 & 58 & $\begin{array}{l}\text { FDG- PET scan: intense } \\
\text { hypermetabolic activity } \\
\quad \text { (SUV 10.5) }\end{array}$ & ND & Normal & ND & ND & $\mathrm{ND} / \mathrm{ND}$ \\
\hline Case 3 & 57 & $\begin{array}{c}\text { Noninflammatory palpable } \\
\text { tumefaction }\end{array}$ & Hypoechoic lesion & Nodular lesion & $\begin{array}{l}\text { T2 enhancement, Hyposignal } \\
\text { T1, gadolinium enhancement }\end{array}$ & ND & $+/ \mathrm{ND}$ \\
\hline
\end{tabular}

*BS: Breast Sarcoidosis, MRI: Magnetic Resonance Imaging, ND: Not Determined 
Table 3. Breast mass in sarcoidosis patients: differential diagnosis.

- Tuberculosis

- Leprosy

- Brucellosis

- Typhoid

- Blastomycosis

- Coccidioidomycosis

- Sporothrichosis

- Histoplasmosis

- Hydatid disease

- Cysticercosis

- Filariasis or oxyuris infestation

- Granulomatous mastitis

- Foreign-body

- Reaction from surgical procedures or substances introduced for cosmetic reasons

were thoracic in $81 \%$ of cases (stage I: 6 cases; stage II: 8 cases; stage III: 4 cases) [5-7,10,12,13,17-21,23-26] (Cases 1, 2 and 3), skin (23\%) $[11,14,18,22]$ (Case 2) and eyes (18\%) [6,20] (Cases 2 and 3). Sarcoidosis was multi-systemic ( $\geq$ to 2 organs and/or hypercalcemia) in $45 \%$ of cases. Nodules, masses, and discomfort of breast revealed BS in $60 \%$ of cases with normal skin appearance [4-7,10-13,15,16-22,25] (Case 3). Usually, physical examination revealed multiple unilateral or bilateral breast masses without skin/nipple changes or lymphadenopathy. Only 1 patient had extensive skin lesion with palpable induration of left breast [25].

BS could also be discovered on systematic echo/mammography (6 cases) [14,21,23,24,26] (Case 1), or FDG-pet scan (Case 2). The echographic lesion appears as an irregular hypoechoic mass (8/9 cases) $[13,18-21,25,26]$ (Case 3), or shows ectasia of mammary duct and dense nodular lesion [26] (Case 1). Bilateral mammography performed in 17 cases was normal in 2 cases [12], (case 2), or demonstrate abnormal pictures in 10 cases such as unique [13,15,17-20,22-25] or multiple irregular and/or spiculated masses [14,21]. Of note, micro calcifications are exceptional [23] as well as dilated duct [26]. MRI was rarely reported but it was always abnormal (4/4), showing irregular mass with gadolinium enhancement $[13,18,19,25]$ (Case 3). FDG-PET-scan can show hypermetabolic breast lesion (as in our Case 2), sometimes associated to sarcoidosis typical butterfly distribution pattern of hypermetabolic mediastinal and hilar lymphadenopathy [27] (Case 2). As all imaging suggest malignancy, breast biopsies are mandatory to eliminate all differential diagnoses including carcinoma (Table 3). In our case 2, once having eliminated differential diagnosis, we estimated appropriate to evoke a breast localization of sarcoidosis as breast hypermetabolism appeared simultaneously to a severe multisystemic flare up of the disease. Regression of hypermetabolism following immunosuppressive treatment confirmed our diagnosis.

In 2 cases, fine needle aspiration cytology demonstrates lymphocytes, epithelioid-like cells, aggregate histiocytes or/and reticulocytes without necrosis $[17,24]$ and/ or core biopsy $(10 / 10$ cases) $[14,15,19,21,22,24,25]$ (Cases 1 and 3), and/or excision (16/16 cases) $[4-7,10,13,15-18,20,23,24]$. In order to eliminate sarcoid-like reaction, clinical, laboratory and morphological investigations (Chest $\mathrm{X}$ ray, CT scan, FDG PET scan) must be consistent with a diagnosis of sarcoidosis. Typical histological pattern of sarcoidosis was noted in other organs than breast in 12 cases [5,6,7,11,13,14,24] (Cases 1, 2 and 3 ) or Kweim test alone $[10,11]$. Based on 14 cases with sufficient details, the mean follow up was 39.42 months (12 to 120). Among the 7 published cases who received steroids for their sarcoidosis, none relapsed from BS. However, in our 3 cases, despite immunosuppressive therapy, we did not observe regression of mammography abnormalities in two cases while we found a normalization of FDG-PET-scan in one case. In other published cases, BS was asymptomatic, with an indolent course and no therapy was required except excision. Breast localization of sarcoidosis was not an indication to immunosuppressive treatment. The latter was indicated for extra-breast severe localization of sarcoidosis which might compromise vital or functional prognosis. In those cases, corticosteroids were usually able to induce clinical and imaging improvement of BS [5,12,13,20,23,24,25] (Cases 1, 2 and 3). When BS is diagnosed, it is necessary to have regular follow up due to the possibility of coexisting BS and breast malignancy [3,5,10,30-32]. Nevertheless, previous reports did not demonstrated any association between sarcoidosis and breast cancer $[9,30]$.

Our three cases report and 22 previously published cases emphasize the possibility to observed BS, independently of breast carcinoma. Breast sarcoidosis remains a rare localization of sarcoidosis with diverse and nonspecific characteristics. Imaging features frequently mimic a breast cancer. Core biopsies are mandatory to confirm sarcoidosis and eliminate other diagnoses. In cases with localized BS, breast mass excision seems to be sufficient with no relapse of BS. Nevertheless, a prolonged breast follow-up is necessary in all cases.

\section{References}

1. Chapelon C, Uzzan B, Piette JC, Jacques C, Coche E, et al. (1984) Sarcoidosis in internal medicine. A cooperative study of 554 cases. Ann Med Interne (Paris) 135: 125-131. [Crossref]

2. Ungprasert P, Carmona EM, Utz JP, Ryu JH, Crowson CS, et al. (2016) Epidemiology of Sarcoidosis 1946-2013: A Population-Based Study. Mayo Clin Proc 91: 183-188. [Crossref]

3. Lower EE, Hawkins HH, Baughman RP (2001) Breast disease in sarcoidosis. Sarcoidosis Vasc Diffuse Lung Dis 18: 301-306. [Crossref]

4. Rigden B (1978) Sarcoid lesion in breast after probable sarcoidosis in lung. Br Med J 2: 1533-1534. [Crossref]

5. Ross MJ, Merino MJ (1985) Sarcoidosis of the breast. Hum Pathol 16: 185-187. [Crossref]

6. Gansler TS, Wheeler JE (1984) Mammary sarcoidosis. Two cases and literature review. Arch Pathol Lab Med 108: 673-675. [Crossref]

7. Fitzgibbons PL, Smiley DF, Kern WH (1985) Sarcoidosis presenting initially as breast mass: report of two cases. Hum Pathol 16: 851-852. [Crossref]

8. Isley LM, Cluver AR, Leddy RJ, Baker MK (2012) Primary sarcoid of the breast with incidental malignancy. J Clin Imaging Sci 2: 46. [Crossref]

9. Chen J, Carter R, Maoz D, Tobar A, Sharon E, et al. (2015) Breast Cancer and Sarcoidosis: Case Series and Review of the Literature. Breast Care (Basel) 10: 137140. [Crossref]

10. Banik S, Bishop PW, Ormerod LP, O'Brien TE (1986) Sarcoidosis of the breast. J Clin Pathol 39: 446-448. [Crossref]

11. McPherson JG, Yeoh CB (1993) Rare manifestations of sarcoidosis. J Natl Med Assoc 85: 869-872. [Crossref] 
12. Donaldson BA, Polynice A, Oluwole S (1995) Sarcoidosis of the breast: case report and chart review. Am Surg 61: 778-780. [Crossref]

13. Kenzel PP, Hadijuana J, Hosten N, Minguillon C, Oellinger H, et al. (1997) Boeck sarcoidosis of the breast: mammographic, ultrasound, and MR findings. $J$ Comput Assist Tomogr 21: 439-441. [Crossref]

14. Gisvold JJ, Crotty TB, Johnson RE (2000) Sarcoidosis presenting as spiculated breast masses. Mayo Clin Proc 75: 293-295. [Crossref]

15. Harris KP, Faliakou EC, Exon DJ, Nasiri N, Gui GP (2000) Isolated sarcoidosis of the breast. J R Soc Med 93: 196-197. [Crossref]

16. Ojeda H, Sardi A, Totoonchie A (2000) Sarcoidosis of the breast: implications for the general surgeon. Am Surg 66: 1144-1148. [Crossref]

17. Takahashi R, Shibuya Y, Shijubo N, Asaishi K, Abe S (2001) Mammary involvement in a patient with sarcoidosis. Intern Med 40: 769-771. [Crossref]

18. Ishimaru K, Isomoto I, Okimoto T, Itoyanagi A, Uetani M (2002) Sarcoidosis of the breast. Eur Radiol 12 Suppl 3: S105-108. [Crossref]

19. El khoury M, Cherel P, Charley H, Bertrand F, Becette V, et al. (2005) Guiz case. Eur J Radiol 54: 2-5.

20. Fiorucci F, Conti V, Lucantoni G, Patrizi A, Fiorucci C, et al. (2006) Sarcoidosis of the breast: a rare case report and a review. Eur Rev Med Pharmacol Sci 10: 47-50. [Crossref]

21. Nicholson BT, Mills SE (2007) Sarcoidosis of the breast: An unusual presentation of a systemic disease. Breast $J$ 13: 99-100. [Crossref]
22. Hermann G, Nagi C, Mester J, Tierstein A (2008) Unusual presentation of sarcoidosis of the breast. Br J Radiol 81: e231-233. [Crossref]

23. Rishi MA, Smith M (2009) Sarcoid of the breast associated with microcalcifications on mammogram. Intern Med J 39: 134-135. [Crossref]

24. Panzacchi R, Gallo C, Fois F, Dalpiaz G, Cucchi MC, et al. (2010) Primary sarcoidosis of the breast: case description and review of the literature. Pathologica 102: 104-107. [Crossref]

25. Zujic PV, Grebic D, Valencic L (2015) Chronic granulomatous inflammation of the breast as a first clinical manifestation of primary sarcoidosis. Breast Care 10: 51-53. [Crossref]

26. Mason C, Yang R, Hamilton R, Punar M, Shah Z, et al. (2017) Diagnosis of sarcoidosis from a biopsy of a dilated mammary duct. Proc (Bayl Univ Med Cent) 30: 197-199. [Crossref]

27. Zivin S, David O, Lu Y (2014) Sarcoidosis mimicking metastatic breast cancer on FDG PET/CT. Intern Med 53: 2555-2556. [Crossref]

28. Akhatari M, Quesada JR, Schwartz MR, Chiang SB, Teh BS (2014) Sarcoidosis presenting as metastatic lymphadenopathy in breast cancer. Clin Breast Cancer 10: e107-e110.

29. Kochoyan T, Akhmedov M, Shabanov A, Terekhov I (2016) Sarcoidosis imitating breast cancer metastasis: a case report and literature review. Cancer Biol Med 13: 396398. [Crossref]

30. Brincker H (1995) Sarcoidosis and malignancy. Chest 108: 1472-1474. [Crossref]

Copyright: $(2018$ Chapelon-Abric C. This is an open-access article distributed under the terms of the Creative Commons Attribution License, which permits unrestricted use, distribution, and reproduction in any medium, provided the original author and source are credited. 